The CMS ECAL Barrel HV system

This article has been downloaded from IOPscience. Please scroll down to see the full text article.

2013 JINST 8 C02039

(http://iopscience.iop.org/1748-0221/8/02/C02039)

View the table of contents for this issue, or go to the journal homepage for more

Download details:

IP Address: 131.215.71.79

The article was downloaded on 11/04/2013 at 15:23

Please note that terms and conditions apply. 
Topical Workshop on Electronics for Particle Physics 2012, 17-21 SEPTEMBER 2012, OXFORD, U.K.

\title{
The CMS ECAL Barrel HV system
}

\section{On behalf of the CMS collaboration}
A. Bartoloni, ${ }^{a}, 1$ S. Baccaro, ${ }^{b}$ L.M. Barone, ${ }^{a}$ F. Cavallari, ${ }^{a}$ I. Dafinei ${ }^{a}$ D. Del Re, ${ }^{a}$
M. Diemoz, ${ }^{a}$ E. Di Marco, ${ }^{c}$ M. Grassi, ${ }^{a}$ E. Longo, ${ }^{a}$ P. Meridiani, ${ }^{a}$ F. Micheli, ${ }^{a}$
G. Organtini, ${ }^{a}$ S. Nourbakhsh, ${ }^{a}$ R. Paramatti, ${ }^{a}$ F. Pellegrino, ${ }^{a}$ S. Rahatlou, ${ }^{a}$
C. Rovelli, ${ }^{a}$ M. Sigamani ${ }^{a}$ and L. Soffi ${ }^{a}$

\author{
${ }^{a}$ INFN ROMA, \\ P.le Aldo Moro 2, 00185 Roma, Italy \\ ${ }^{b}$ ENEA - Casaccia Research Center, \\ Via Anguillarese 301, 00123 Roma, Italy \\ ${ }^{c}$ California Institute of Technology, \\ 1201 East California Boulevard, Pasadena, CA 91125, U.S.A. \\ E-mail: alessandro.bartoloni@roma1.infn.it
}

\begin{abstract}
The CMS electromagnetic calorimeter (ECAL) comprises 75848 scintillating lead tungstate crystals. 61200 crystals are contained in the ECAL Barrel section and are read out by avalanche photodiode (APD) with internal gain of about 50. This gain is achieved with a high voltage (HV) of about 400 Volts. The gain stability requirement implies a supply voltage stable to within $0.01 \%$. We describe our experience with the installed Barrel HV power supply system, which has been used for data taking since 2008 .
\end{abstract}

KEYWORDS: Voltage distributions; Calorimeters

\footnotetext{
${ }^{1}$ Corresponding author.
} 


\section{Contents}

1 The CMS ECAL Barrel HV system 1

1.1 The CMS ECAL barrel 1

$\begin{array}{ll}1.2 \text { HV requirements } & 2 \\ 1.3 & \text { The HV system }\end{array}$

1.3 The HV system 3

2 The ECAL Barrel HV system performance during the first 3 years of operation in CMS

2.1 HV stability

2.2 APD Dark Current increase 6

$\begin{array}{llr}3 & \text { Conclusion } & 8\end{array}$

\section{The CMS ECAL Barrel HV system}

The Electromagnetic Calorimeter (ECAL) used in the CMS [1] experiment at the LHC uses scintillating crystals to detect and measure the energy of photons and electrons produced in the collisions. The light produced is read out in the barrel part by Avalanche Photodiodes (APD) that needed to be appropriately biased to a voltage of about 400 Volts with high stability and ultra low noise figures. In 1998, when the INFN Roma group took the responsibility to develop such ECAL subsystem, the required electrical performance was present in "state of the art" laboratory power supplies but it was not possible, using such devices, to build in a reliable way a large scale system as required to bias 122400 APDs. For this reason it was necessary to develop a dedicated high voltage system with the required electrical specification for the output voltage and current, and engineered to host $1124 \mathrm{HV}$ channels, each with the possibility to be controlled and monitored remotely through Ethernet connection.

\subsection{The CMS ECAL barrel}

The barrel part of CMS ECAL (see figure 1) comprises 61200 lead tungstate $\left(\mathrm{PbWO}_{4}\right)$ crystals whose scintillation light is detected using APDs produced by Hamamatsu Photonics [2, 3]. Two APDs are used for each crystal (see figure 2). A dedicated high voltage (HV) power supply system is used to bias the APDs. The performance of the calorimeter can be described in terms of the energy resolution expressed as a function of the incident electron/photon energy by the following formula:

$$
\frac{\sigma_{E}}{E}=\frac{a}{\sqrt{E}} \oplus \frac{b}{E} \oplus c
$$

where the first is the stochastic term, the second is the electronic noise term and the third is the so-called constant term, which includes contributions from calibrations and instabilities. Possible APD gain stability will contribute to the constant term, since the gain of the APDs depend from HV stability, the HV system characteristics directly influence the ECAL energy resolution. 


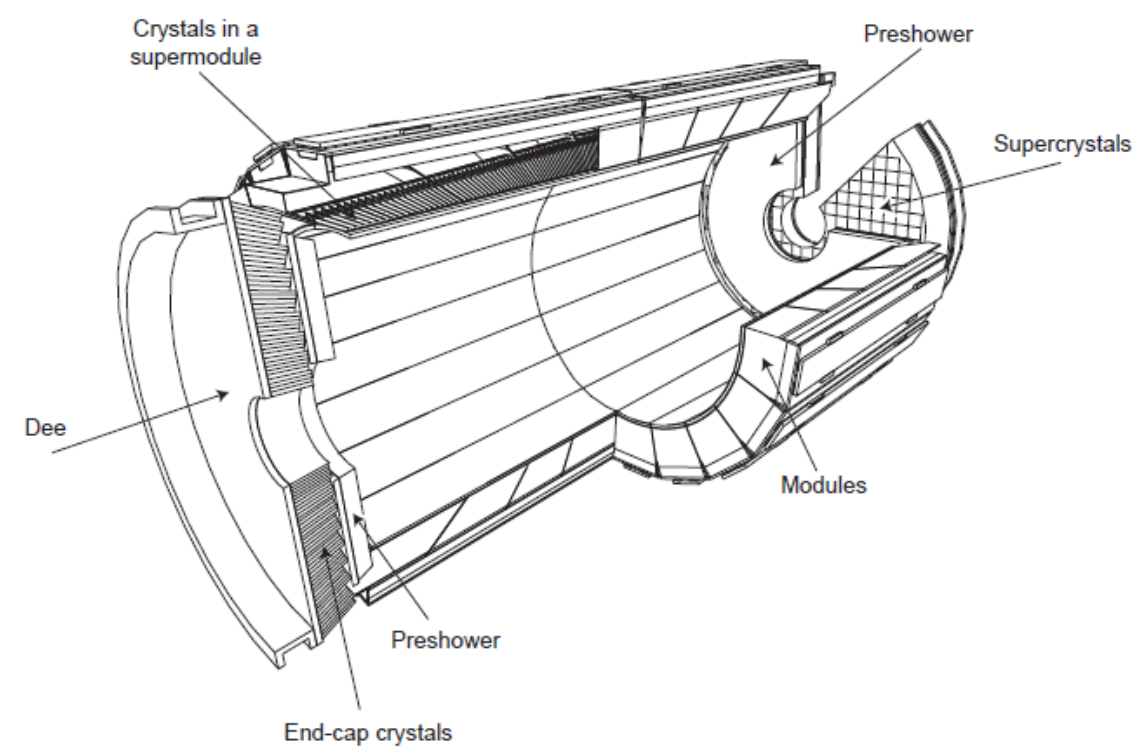

Figure 1. The CMS Electromagnetic Calorimeter (ECAL). The barrel section comprises 36 supermodule, each containing 4 modules.

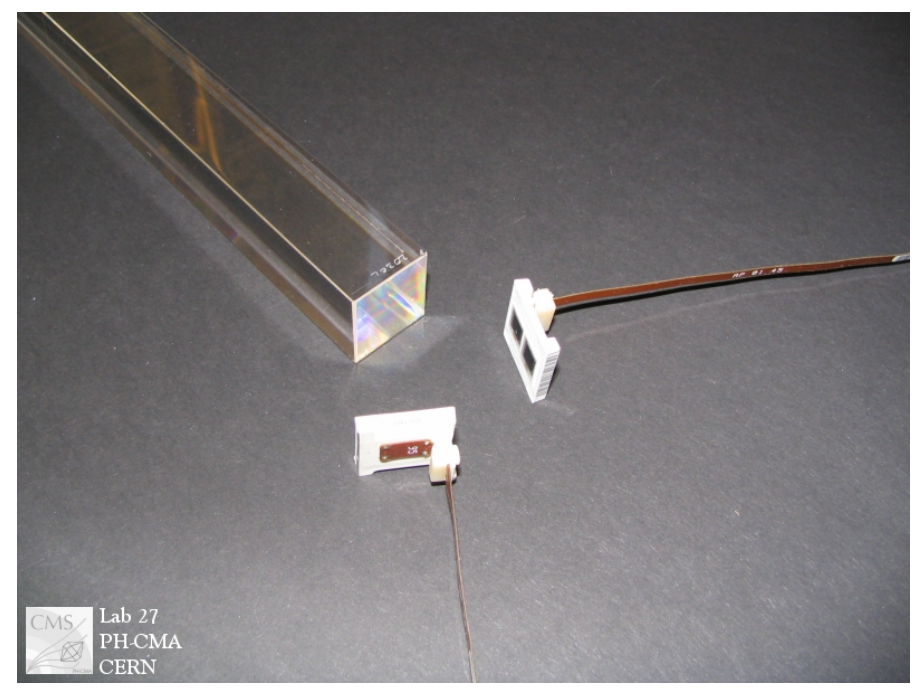

Figure 2. A crystal $\left(\mathrm{PbWO}_{4}\right)$ used in the CMS ECAL with a "capsule" hosting two Avalanche Photodiodes (APD).

\subsection{HV requirements}

The APDs (see figure 3 and table 1) in CMS are operated at a gain 50, requiring a high (bias) voltage in the proximity of the breakdown region (350-450 Volts).

The APD gain variation is about 3.1\%/Volts at gain 50 and the contribution of this gain variation to the ECAL energy resolution constant term is required to be less than $0.2 \%$. This implies that the high voltage stability has to be of the order of $60-65 \mathrm{mV}$.

This requirement places constraints on the combination of electrical system characteristics including noise, ripple, voltage regulation and absolute precision, for short and long-term periods. 


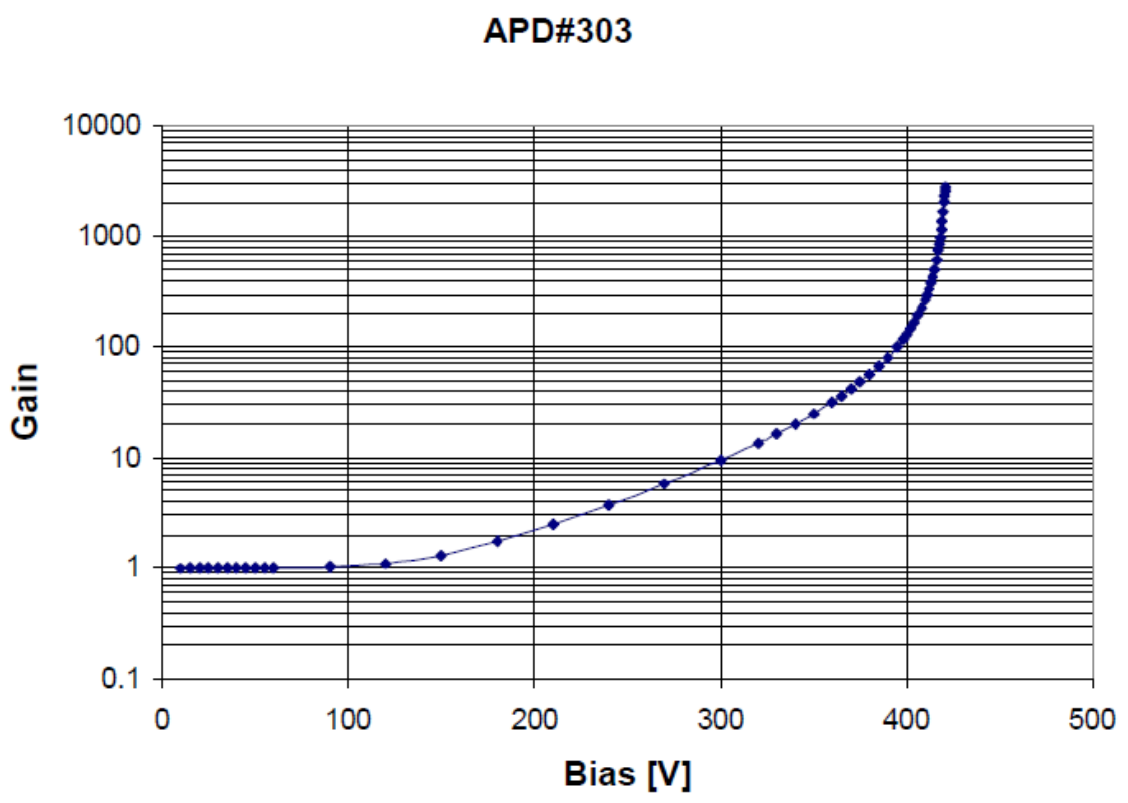

Figure 3. Typical APD gain vs. Bias Curve.

Table 1. APD characteristics and numbers.

\begin{tabular}{|l|c|}
\hline \multicolumn{2}{|c|}{ Parameter } \\
\hline Maximum operating voltage & $500 \mathrm{~V}$ \\
\hline Minimum operating voltage & $200 \mathrm{~V}$ \\
\hline Leakage current (start of experiment) & $<0.01 \mu \mathrm{A}$ \\
\hline Leakage current (after 10 years) & $<20 \mu \mathrm{A}$ \\
\hline dM/dV gain sensitivity (at gain $\mathrm{M}=50)$ & $3.1 \% / \mathrm{V}$ \\
\hline APDs used in the ECAL barrel & 122400 \\
\hline
\end{tabular}

\subsection{The HV system}

The CMS ECAL HV power supply system was developed starting from 1999 by INFN Roma group in collaboration with CAEN Company ${ }^{1}[4,5]$. The system was installed in 2008 in 6 racks in the CMS Underground Service Cavern (USC) (see figure 4) where no damage to the electronics circuits due to radiation is foreseen.

It is composed of 18 CAEN SY1527 mainframes, hosting 144 A1520E modules for a total of 1224 HV channels.

Since APDs are sorted to have similar Voltage bias (Vbias) for gain 50, each HV channel is used to bias 100 APDs (50 capsules and related crystals). Sense wires are used to compensate cable voltage drop. Each capsule receives the bias voltage through a passive filter network and a protection resistor (of $136 \mathrm{kOhm}$ ) to avoid losing all the APDs sharing the same HV channel, in case of a short circuit between one APD cathode and the HV ground (see figure 5).

\footnotetext{
${ }^{1}$ CAEN Viareggio www.caen.it.
} 


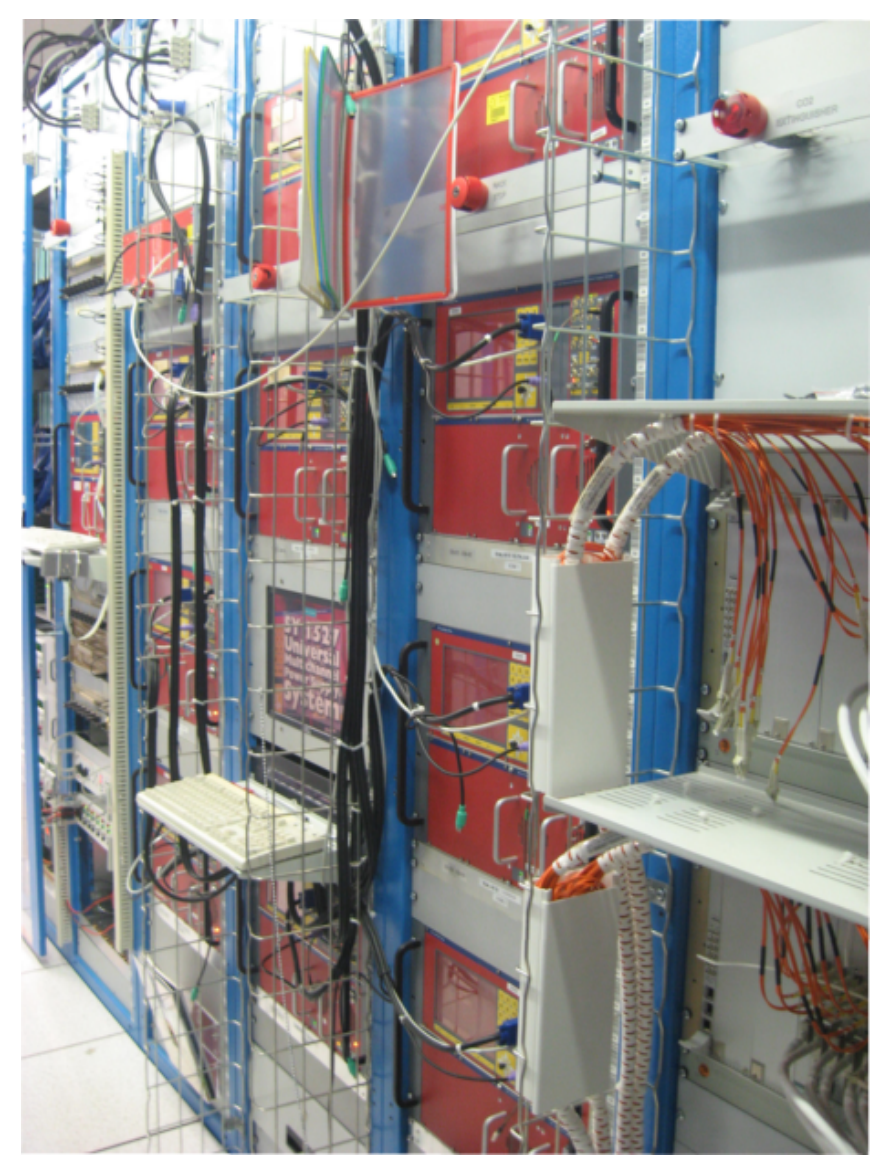

Figure 4. Half of the CMS ECAL Barrel HV system in the USC.
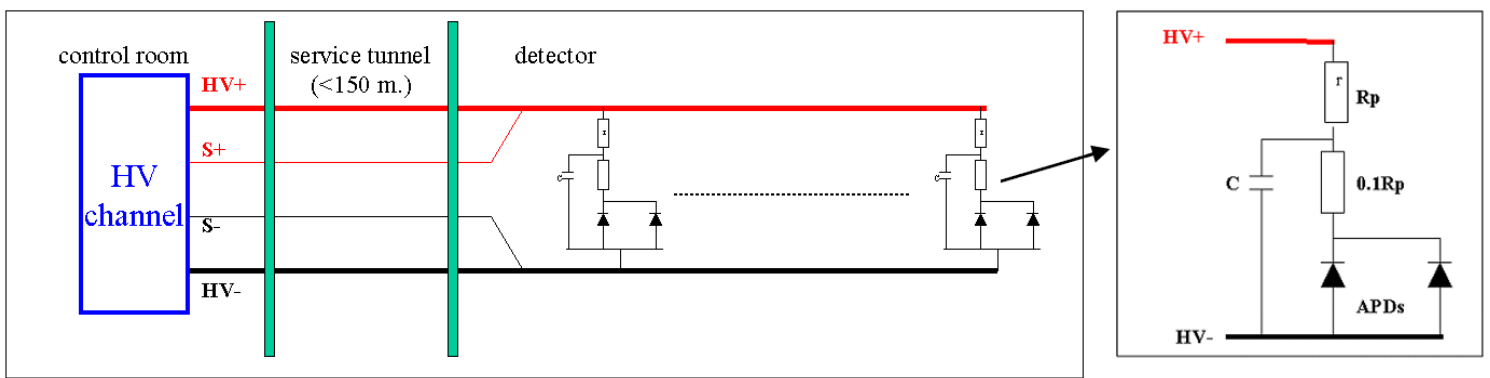

Figure 5. CMS-ECAL APD power supply architecture. 50 capsules (each containing 2 APDs) share the same HV channel. They receive the bias voltage through a protection resistor (Rp) and a RC filter network.

It is possible to set the output voltage in the range $0 \mathrm{~V}-500 \mathrm{~V}$ with a maximum output current of $15 \mathrm{~mA}$ per channel (see table 2).

During the CMS beam test activities performed in 2004 and 2006, the compliance of the HV system to the performance requested by the ECAL energy resolution was proven [6].

Before installation in CMS each channel was tested [5] in a dedicated test-bench. Channels not compliant with the required $65 \mathrm{mV}$ stability over 30 days were not used (see figure 6). 
Table 2. HV Channel electrical characteristics.

\begin{tabular}{|l|c|}
\hline \multicolumn{1}{|c|}{ Parameters } & \\
\hline Output voltage range & $0-500 \mathrm{~V}$ \\
\hline Programmable setting step & $20 \mathrm{mV}$ \\
\hline DC regulation at load & $< \pm 20 \mathrm{mV}$ \\
\hline DC stability at load (over 90 days) & $< \pm 20 \mathrm{mV}$ \\
\hline Low freq. noise at load (f $<100 \mathrm{kHz})$ & $< \pm 20 \mathrm{mV}$ \\
\hline High freq. noise at load (f $>100 \mathrm{kHz})$ & $< \pm 20 \mathrm{mV}$ \\
\hline Operating temperature at supply & $15 \div 40^{\circ} \mathrm{C}$ \\
\hline Current limit & $15 \mathrm{~mA}$ \\
\hline On and off maximum ramp rate & $50 \mathrm{~V} / \mathrm{sec}$. \\
\hline External calibration & $< \pm 20 \mathrm{mV}$ \\
\hline
\end{tabular}

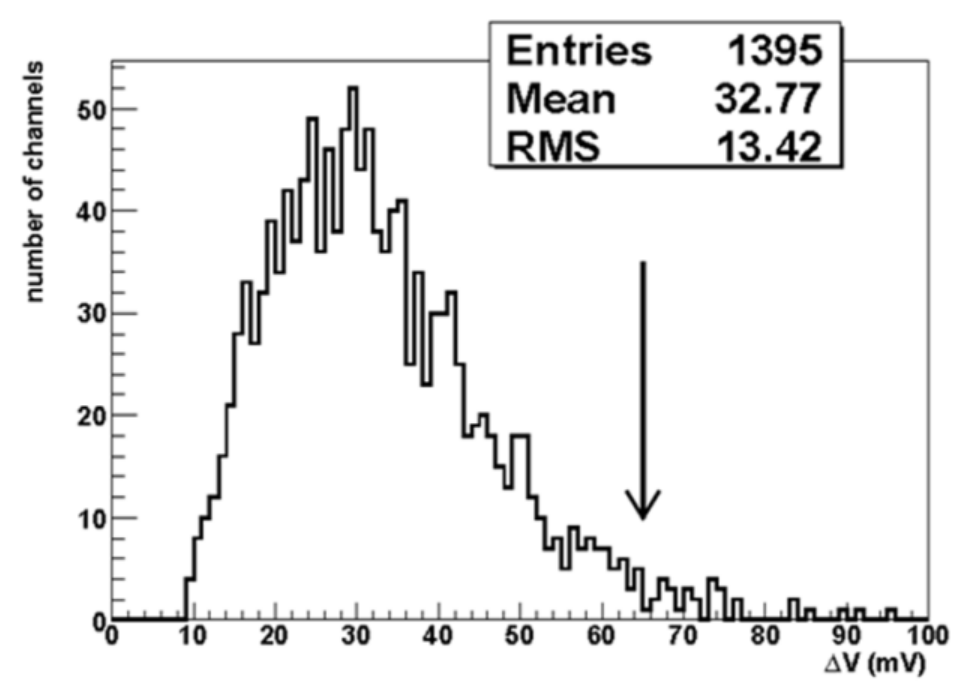

Figure 6. HV Channels stability test result. Absolute values of the measured deviations from the desired output voltage at the end of the test (30 days) are shown for all the channels under test. HV channels with $\mathrm{dV}>65 \mathrm{mV}$ were not used.

\section{The ECAL Barrel HV system performance during the first 3 years of operation in CMS}

Since the very beginning, during the commissioning phase [7], and then during operation with beams, the HV system was monitored and controlled (reading and setting of all individual channel parameters: status, output voltage, output current, etc.) by the ECAL Detector Control System (DCS) over Ethernet. The DCS continuously checks critical parameters, generating warnings and alarms to the CMS control room and to the ECAL experts if necessary.

A team of $\mathrm{HV}$ experts, who are on-call during CMS operation, promptly repairs failures and provides routine maintenance. 


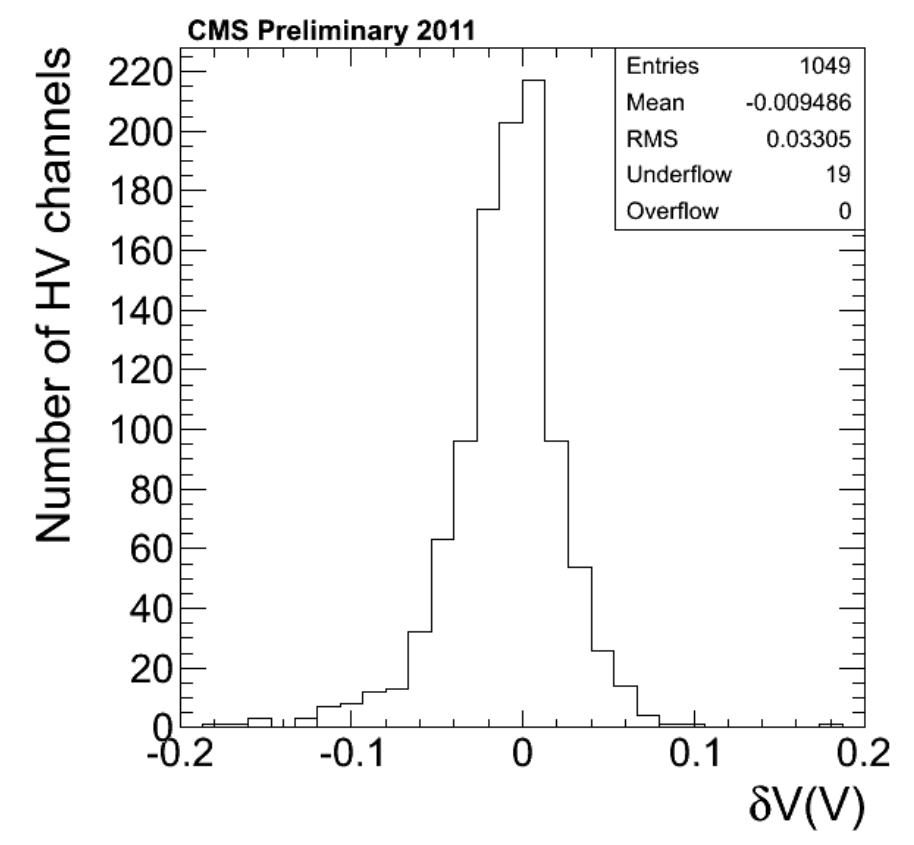

Figure 7. HV Channels voltage stability during 2011 run. In the plot the dV channel distribution is shown.

Periodically, during the LHC winter stop, a calibration of all channels is performed using a dedicated external system, to guarantee an absolute voltage precision with an accuracy of $\pm 20 \mathrm{mV}$.

\subsection{HV stability}

During the past three years the HV system stability has been measured using data taken during the periodical calibration.

The HV system is calibrated once or twice a year with dedicated electronics that allows us, before the calibration of each channel, to measure the output voltage deviation at 380 Volt using a $61 / 2$ digit digital multi-meter.

Using calibration data taken at the beginning of the 2012, the output voltage deviations during 2011 are estimated to be $33 \mathrm{mV}$ (RMS) as shown in figure 7.

Taking into account the gain dependence of APD gain from bias voltage of:

$$
\delta \text { Gain } / \text { Gain }=\beta \delta V \quad \beta=3.1 \% / \text { Volt }(\text { at gain } 50)
$$

an estimate has been made of the effective APD gain stability in 2011. The estimate includes partial corrections to changes in gain from measurements from the ECAL laser monitoring system that are incorporated in the response corrections to data.

Such measurements show excellent performance, corresponding to an APD gain stability of better than $0.2 \%$ for $>97 \%$ of channels (see figure 8 ).

\subsection{APD Dark Current increase}

The DCS monitors the APD dark current evolution due to the radiation damage (see figure 9). Different values of pseudo-rapidity for APDs imply different value of neutron radiation dose and 


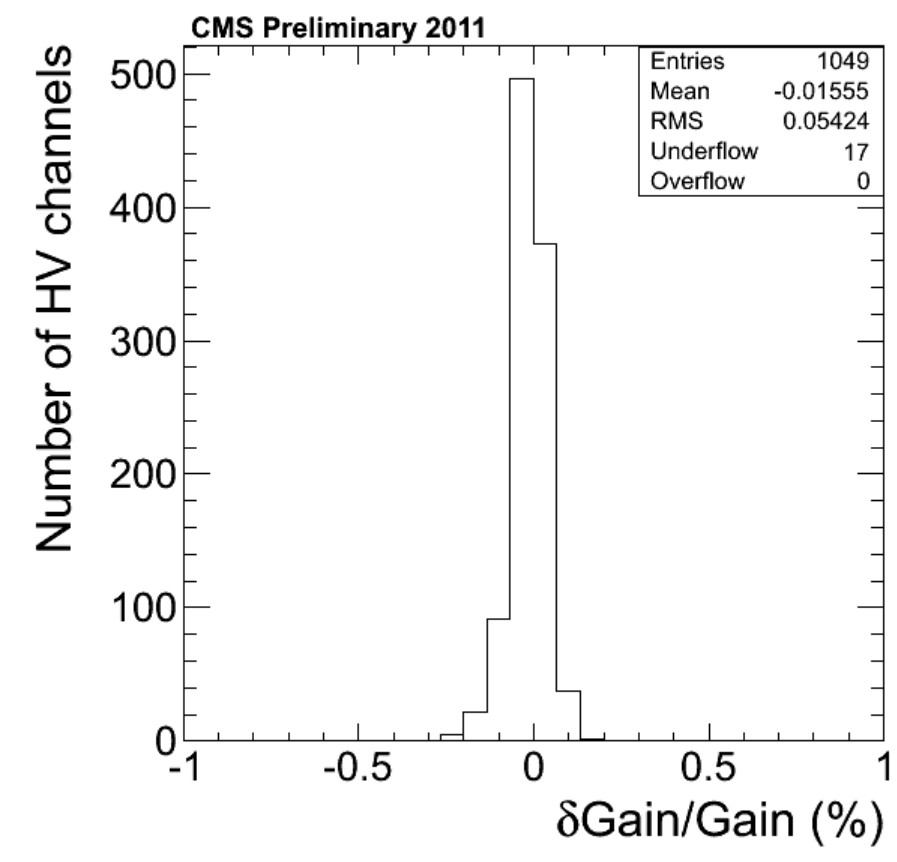

Figure 8. The gain deviation for all HV channels measured after one year of data taking (2011). These gain instabilities due to the APD HV are at the $0.05 \%$ level after correction via the laser system.

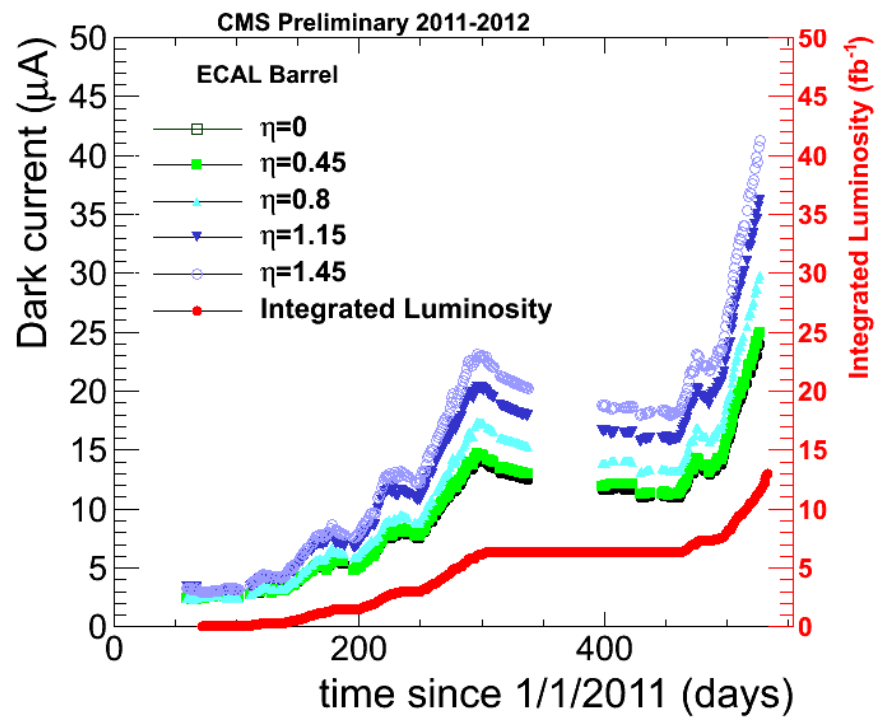

Figure 9. The plot shows the APD dark current (for $1 \mathrm{HV}$ Channel = 100 APDs) increase during the 2011 \& 2012 runs and corresponding integrated luminosity (red points). The different blue/green colors represent the channels located at different pseudo-rapidity in one ECAL Supermodule (1700 crystals).

hence of increase in dark current (dependence from $\eta$ in figure 9). Recovery from damage can be observed from the decrease in dark current during the LHC winter stop.

The leakage currents are consistent with the expectations from the system design phase corresponding to a maximum current per $\mathrm{HV}$ channel of $<2 \mathrm{~mA}$ after $500 \mathrm{fb}^{-1}$ integrated luminosity. 


\section{Conclusion}

The High Voltage system developed for the barrel part of the CMS ECAL has been described in this paper. The HV system has operated successfully during the first 3 years of operation in CMS. The APD gain stability is better than $0.2 \%$ for $97 \%$ of all channels, which meets the specification required for limiting the impact on the ECAL energy resolution. The increase in APD dark current is in line with expectations.

\section{Acknowledgments}

We thank all the people that supported us during these 14 years of activities especially those of CAEN Company. We also thank Ing. Stefano Petrucci, designer of the A1520E module used in the system, for his crucial and continuous support to our work.

\section{References}

[1] CMS collaboration, The CMS experiment at LHC CERN, 2008 JINST 3 S08004.

[2] S. Baccaro et al., Radiation damage effect on avalanche photodiode, Nucl. Instrum. Meth. A 426 (1999) 206.

[3] Z. Antunovic et al., Radiation hard avalanche photodiode for the CMS detector, Nucl. Instrum. Meth. A 537 (2005) 379.

[4] A. Bartoloni et al, High voltage system for the CMS Electromagnetic Calorimeter, Nucl. Instrum. Meth. A 582 (2007) 462.

[5] A. Bartoloni, The power supply system for CMS ECAL APDs, in Proceedings of the 7th Workshop on Electronics for LHC Experiments, Stockholm Sweden, 10-14 Sep 2001, pp. 358-362.

[6] P. Adzic et al., Energy resolution of the barrel of the CMS Electromagnetic Calorimeter, 2007 JINST 2 P04004.

[7] CMS collaboration, Performance and operations of the CMS Electromagnetic Calorimeter, 2010 JINST 5 T03010. 